\title{
Associations between Notch-2, Akt-1 and HER2/neu Expression in Invasive Human Breast Cancer: A Tissue Microarray Immunophenotypic Analysis on 98 Patients
}

\author{
Ada Maria Florena Claudio Tripodo Carla Guarnotta Sabrina Ingrao \\ Rossana Porcasi Anna Martorana Giosuè Lo Bosco Daniela Cabibi \\ Vito Franco \\ Dipartimento di Patologia Umana, Università degli Studi di Palermo, Palermo, Italia
}

\section{Key Words \\ Breast cancer • Tissue microarray $\cdot$ HER2 $\cdot$ Akt $\cdot$ Notch}

\begin{abstract}
Objective: We aimed to investigate the existence of associations between well-established and newly recognized biological and phenotypic features of breast cancer involved in tumor progression and prognosis. Methods: Ninety-eight cases of invasive breast cancer were assessed for the immunohistochemical expression of estrogen and progesterone receptors, Ki-67, HER2, Akt-1, and Notch-2, using the tissue microarray technique. Data regarding tumor histotype, histological grade, tumor size and lymph node status were collected for each patient and included in the analysis. Results: Several significant associations between histological and/or immunophenotypic features came from the analysis of our data. Positive associations were observed between estrogen and progesterone receptors, tumor grade and proliferation index, tumor grade and HER2, Akt-1 and estrogen receptors, and Notch-2 and HER2. Inverse associations were noted between hormone receptors and tumor grade, hormone receptors and HER2, Akt-1 and tumor grade, and Akt-1 and nodal invasion. Conclusions: Our results, showing the existence of a number of estrogen receptor-positive tumors with
\end{abstract}

Akt-1 expression, better degree of differentiation, and no lymph node involvement, along with the presence of HER2positive tumors with strong Notch-2 expression, support the role of Notch and Akt in breast cancer progression and suggest that they may also represent new appealing therapeutic targets.

Copyright $\odot 2007$ S. Karger AG, Basel

\section{Introduction}

Breast cancer is the most common cancer in women, accounting for about one third of all cancers. As for most epithelial malignancies, the incidence of breast cancer shows a straight-line increase with every year of life, with the chance of developing breast cancer of about $1.4 \%$ in the 5 th decade rising to 3.6 after the age of 60 years [1]. In addition to ageing, a number of breast cancer-predisposing factors have been described so far. Among these factors, a few, such as hormones, fared better in assessing risk and prognosis and eventually proved to be good substrates for the development of targeted therapies. As breast cancer is a hormone-dependent disease, the role of estrogens in tumor initiation and promotion has been extensively investigated, highlighting the importance of the receptor status

Dr. Claudio Tripodo

Dipartimento di Patologia Umana, Università degli Studi

Via del Vespro 129

IT-90127 Palermo (Italy)

E-Mail karger@karger.ch_Accessible online at:

www.karger.com www.karger.com/pat 
of breast cancer cells and introducing successful therapies such as tamoxifen and aromatase inhibitors [2].

Recently, the identification of a noticeable frequency of selected oncogene amplifications, namely HER2/neu, C-MYC, CCND1, has drawn much attention to the regulation and cross talk of complex signaling pathways in human breast cancer [2]. An ever-increasing number of molecules are being investigated, which play key roles in cell survival and differentiation and that are shared between multiple signaling pathways. Among them, Akt and its functional neighbors act on stimulation of growth and proliferation, inhibition of cell death, or, most often, a combination of these mechanisms [3].

Notch proteins modulate differentiation, proliferation, and apoptotic programs and the demonstration that several human diseases result from mutations in genes encoding Notch receptors or their ligands has validated the Notch signaling pathway as a potential therapeutic target [4].

The recent development of an array-based technique (tissue microarray, TMA) has provided the opportunity to investigate hundreds of molecular markers in the same set of specimens $[5,6]$. This technique allows a maximization of tissue resources and makes possible large scale retrospective studies using formalin-fixed, paraffin-embedded small-core biopsies placed in the same block.

In the present study we aimed to investigate an exploratory hypothesis dealing with the possible associations between multiple biological features that can be involved in breast cancer progression and prognosis, by the histological and immunophenotypic analysis of 98 cases of human breast cancer using TMA.

\section{Methods}

\section{Tissue Samples and Microarrays}

Between January 2003 and February 2004, 98 consecutive cases of invasive breast cancer were selected for review from the archives of the Institute of Pathology of the University of Palermo. Tissue samples had been fixed in $4 \%$ buffered formaldehyde ( $\mathrm{pH} 7.0)$ and paraffin embedded. Lymph node samples (47 axillary dissectomies and 38 sentinel biopsies) were available in 85 out of 98 cases; in 13 cases information on lymph node status was missing. Patients' clinical history and tumor characteristics including tumor histotype, histological grade, tumor size and lymph node status were collected from archival data. All the diagnoses had originally been based on the analysis of hematoxylin-eosin (HE)-stained slides followed by immunohistochemical evaluation. As far as neuroendocrine carcinoma was concerned the diagnosis was made on the ground of suggestive morphology along with immunophenotypic positivity to chromogranin, synaptophysin and NSE. Tumor grading had been assessed using the Scarff-Bloom-Richardson grading system modified by Elston and Ellis [7]. A histopathological reevaluation was performed to confirm the tumor histotype and grading as well as the neoplastic infiltration of lymph nodes. On reevaluation, the most representative areas of the tumors were identified on HEstained sections in order to produce a breast cancer TMA. Two cylinders of tissue, $0.6 \mathrm{~mm}$ in diameter, were removed from each of the 98 paraffin-embedded samples and placed into a single recipient block. This allowed for the simultaneous immunopheotypic analysis of the 98 cases on a single slide.

\section{Immunophenotypic Evaluation}

Immunohistochemistry was performed on TMA slides to study the expression of a number of biological markers that are involved in tumor progression or that play a role in the assessment of prognosis. For this purpose the following primary antibodies were selected: estrogen receptor (MAb 1D5, BioGenex, dilution 1:20); progesterone receptor (MAb 1A6, BioGenex, dilution 1:20); Ki-67 (MAb MIB-1, DakoCytomation, dilution 1:80); HER2 (Hercep test, DakoCytomation); Akt-1 (MAb 2H10, Cell Signaling, dilution 1:50), and Notch-2 (MAb 25-255, Santa Cruz Biotechnology, dilution 1:100). Immunostainings were carried out by means of the streptavidin-biotin complex method using a panel of antibodies effective on formalin-fixed, paraffin-embedded sections. The slides were microwave treated in $10 \mathrm{~mm}$ Tris/EDTA buffer, $\mathrm{pH} 9$ (Target Retrieval Solution, pH 9, 10×, DakoCytomation), for a total of 20 min for antigen unmasking. Endogenous peroxidase activity was blocked by incubation for 10 min with $3 \% \mathrm{H}_{2} \mathrm{O}_{2}$. Sections were subsequently incubated in the presence of a primary antibody overnight at $4^{\circ} \mathrm{C}$. Normal mouse serum was used instead of primary antibodies as a negative control. The specimens were then incubated with biotinylated link antibody and peroxidaselabeled streptavidin (Universal DakoCytomation LSAB + SystemHRP). Staining was completed by incubation with AEC (3-amino9-ethylcarbazole) substrate-chromogen (DakoCytomation AEC Substrate Chromogen Ready-to-Use). An immunophenotypic evaluation was performed independently by two of the authors (V.F., A.M.F.). Estrogen and progesterone receptor expression was reported by a dichotomous variable (0.1) and, owing to their high intratumoral variability, an average of $\geq 10 \%$ reactive neoplastic cells was considered as a positive reaction. HER 2 was graded according to the Dako scoring system. Akt-1 and Notch-2 expressions were scored in a semiquantitative fashion $(-,+,++,+++)$ depending on the staining intensity and the percentage of the positive cells. The proliferation index was assessed by counting the number of Ki-67-positive cells per high-power microscopic field (400×; $0.5 \mathrm{~mm}$ diameter) and was expressed as a percentage. All the morphological and immunophenotypic evaluations were performed under a Nikon Eclipse 80i optical microscope.

\section{Statistical Analysis}

In order to perform the statistical analysis, a set of 11 variables was considered which included pure categorical (tumor histotype, lymph node status, estrogen receptors, progesterone receptors), ordinal (histological grade, HER2, Akt-1, Notch-2), and continuous variables (age on diagnosis, MIB-1 and primary tumor size). The continuous variables were scaled into ordinal categories according to proper threshold values as follows: age: $<40, \geq 40<60$, $\geq 60<70, \geq 70$ years; tumor size: $<1, \geq 1<3, \geq 3<5, \geq 5 \mathrm{~cm}$; Ki- $67:<10, \geq 10<40, \geq 40 \%$. A $\chi^{2}$ test was performed for each possible couple of the 11 selected variables. The $\chi^{2}$ test was calculated 
Table 1. Frequencies and percentage of pathological features of the 98 cases (histological tumor types, tumor grades and lymph node metastasis)

\begin{tabular}{llc}
\hline & & Number \\
\hline Tumor type & ductal & $65(66)$ \\
& lobular & $27(27.5)$ \\
& tubular & $2(2)$ \\
& papillary & $1(1)$ \\
& sarcomatoid & $1(1)$ \\
& gelatinous & $1(1)$ \\
medullary & $1(1)$ \\
Second histotype & ductal & $4(17.4)$ \\
& lobular & $6(26)$ \\
gelatinous & $3(13)$ \\
Grade & nubular & $3(13)$ \\
& neuroendocrine & $7(30.4)$ \\
Lymph node & 1 & $23(23.5)$ \\
& 2 & $54(55)$ \\
& 3 & $21(21.4)$ \\
& positive & $39(40)$ \\
& negative & $46(47)$ \\
& not available & 13 \\
\hline
\end{tabular}

Figures in parentheses represent percentage.
Table 2. Results of the immunophenotypic evaluation on tissue microarray

\begin{tabular}{llc}
\hline & & Number \\
\hline ER expression & positive & $71(72.5)$ \\
& negative & $27(27.5)$ \\
PR expression & positive & $63(64.3)$ \\
& negative & $35(34.7)$ \\
HER2 score & $-/+$ & $16(16.3)$ \\
& ++ & $11(11.2)$ \\
Akt-1 score & +++ & $8(8.2)$ \\
& - & 48 \\
& + & $14(14.3)$ \\
Notch-2 score & ++ & $23(23.5)$ \\
& +++ & $13(13.3)$ \\
& - & $32(32.6)$ \\
& + & $46(47)$ \\
& ++ & $11(11.2)$ \\
& +++ & $9(9.2)$ \\
\hline
\end{tabular}

Figures in parentheses represent percentage. ER = Estrogen receptor; $\mathrm{PR}=$ progesterone receptor. in a standard way when the variables were both pure categorical; otherwise, if there was at least one ordinal variable, only those groupings between the ordinal variable categories that were considered sound were included in the calculation. In this latter case we considered the variables as associated when at least one grouping that was sound showed a significant association, verifying also that in the contingency table $80 \%$ of the cells had expected frequencies greater than 5 and that all the cells had expected frequencies greater than 1 . Fifty-five single statistical tests were performed.

This method allowed us to perform an in-depth study of the associations between the above-mentioned variables. A minimum p value of 0.05 was considered to confirm associations with a probability of $95 \%$ [8]. Due to the exploratory character of the study, no $\mathrm{p}$ value correction was done.

\section{Results}

All the specimens collected for the study were from female patients aged $34-88$ years (median age 66 ; minimum 34 , maximum 88). On histopathological revision all the 98 diagnoses of invasive breast cancer were confirmed. All the histological data are detailed in table 1 . The tumors were divided according to their histotype as follows: 65 infiltrating ductal carcinomas, 27 infiltrating lobular, 2 tubular, 1 medullary, 1 papillary, 1 sarcomatoid, and 1 gelatinous; 23 cases showed also a second histotype, namely infiltrating ductal cancer in 4 cases, infiltrating lobular cancer in 6 , gelatinous cancer in 3 , tubular cancer in 3 , and neuroendocrine cancer in 7 . Of these 23 cases, only the primary histotype was included in the TMA. Twenty-three cases were grade one, 54 were grade two, and 21 were grade three. Of the 85 cases in which lymph node samples were available, 39 showed the presence of metastatic colonization while 46 were not involved.

The results of the immunophenotypic evaluation of TMA are reported in table 2. Overall, estrogen and progesterone receptors were both expressed in 63 tumors while estrogen receptors alone were expressed in 8 cases. A significant expression of HER2 $(++,+++)$ was observed in 19 cases, 11 of which were scored ++ and 8 were scored +++ . Akt-1 was expressed in 50 cases, its expression in malignant cells ranging from a mild (+) to strong $(+++)$ cytoplasmic staining. Notch-2 proved to be expressed in a rather high percentage of breast cancers (67\%) giving a cytoplasmic staining pattern; its positivity was considered significant $(++,+++)$ only in 18 out of 98 cases.

On statistical analysis several associations between histological and/or immunophenotypic parameters were found and the results are summarized in table 3.

Significant positive associations were observed between estrogen and progesterone receptors, tumor grade 



Fig. 1. a Akt-1 (+++) is intensively expressed in a case of HER2negative infiltrating ductal carcinoma G1. b Notch-2 (++) and HER2 $(+++)$ expressions are nicely associated in this case of infiltrating ductal carcinoma G2.

Table 3. Significant associations between the studied variables

\begin{tabular}{llllr}
\hline Variable 1 & Variable 2 & Association & p value & $\chi^{2}$ \\
\hline ER & PR & + & 0.002 & 39.19 \\
ER & grade & - & 0.02 & 4.82 \\
PR & grade & - & 0.03 & 4.36 \\
Grade & MIB-1 & + & 0.008 & 5.53 \\
ER & HER2 & - & 0.02 & 4.33 \\
HER2 & grade & + & 0.005 & 6.02 \\
HER2 & MIB-1 & + & 0.01 & 7.41 \\
Akt-1 & ER & + & 0.02 & 3.89 \\
Akt-1 & grade & - & 0.04 & 4.20 \\
Akt-1 & HER2 & - & 0.03 & 6.67 \\
Akt-1 & LN & - & 0.02 & 4.23 \\
Notch-2 & HER2 & + & 0.004 & 4.08 \\
\hline
\end{tabular}

$\mathrm{ER}=$ Estrogen receptors; $\mathrm{PR}=$ progesterone receptors; $\mathrm{LN}=$ lymph node status; + = direct association; - = inverse association. and proliferation index, and tumor grade and HER2. Inverse associations were noted between hormone receptors and tumor grade, and hormone receptors and HER2.

Akt-1 proved to be negatively associated with the tumor grade, its expression being strong in well-differentiated tumors and reduced in high-grade ones $(\mathrm{p}=0.02$, fig. 1a), while it showed a direct correlation with estrogen receptors. Akt-1 expression was also associated with the presence of nodal metastases in an inverse fashion ( $\mathrm{p}=$ $0.02)$.

Notch-2 showed a positive association with HER2 $(\mathrm{p}=0.004$, fig. $1 \mathrm{~b})$ although it showed no significant association with tumor grade or hormone receptor status.

\section{Discussion}

The study of Akt and Notch protein families, both involved in the orchestration of intracellular signaling, has followed a rather unusual development in which functional studies occurred prior to and overshadowed those regarding the expression of Notch and Akt proteins in neoplastic diseases. The activation of the Akt pathway has been reported to provide cells with a survival signal that allows them to withstand apoptotic stimuli. In breast cancer, Akt phosphorylation has been correlated with HER2 overexpression and is considered an effector of HER2-induced blockage of tamoxifen action by an active interplay between PI3K and Akt signaling and the estrogen receptor [9]. Aberrant Notch signaling has been observed in a number of human cancers suggesting a possible general role of Notch signaling in tumor formation by causing cells to adopt a proliferative cell fate [10]. It has also been reported that unregulated Notch signaling prevents terminal differentiation of mammary epithelial cells. Aberrant Notch signaling could keep cells in a proliferative state leading to duct dysplasia with an increased risk of progression to carcinoma [10]. Recently, the identification of drugs such as $\gamma$-secretase inhibitors [11] and Ly900/ mTOR inhibitor rapamycin $[12,13]$ able to interfere, respectively, with Notch signaling and PI3/Akt-related pathways has drawn much interest to the opportunity to investigate their role as potential targets for new adjuvant therapies. In particular, the promising effects of therapies combining HER2 inhibitors (gefitinib) or tamoxifen and rapamycin have been recently published $[14,15]$.

On this basis we studied the immunophenotypic expression of these two molecules along with that of wellestablished breast cancer markers and analyzed the relative associations. 
In order to obtain a thorough standardization, a large number of invasive breast cancers were evaluated by the TMA technique that allowed a homogeneous interpretation of the immunohistochemical results and the adoption of a semiquantitative scoring system.

TMA is a method of harvesting small disks of tissue from a range of standard histological sections and placing them in an array on a recipient paraffin block so as to analyze them simultaneously [16]. As TMA analysis facilitates studies of the clinical significance of new genes discovered in genomic screenings of model systems, they have been extensively used to study gene targets. It is also possible to use this technique to associate molecular alterations with a specific stage of tumor progression [17].

Some of the direct or inverse associations resulting from the analysis of our results, such as those between estrogen and progesterone receptors, tumor grade, HER2, and proliferation index, have already been reported in the literature and extensively studied [2]. In a recent paper the association of estrogen receptor, progesterone receptor, bcl-2, HER2/neu, p53 and Ki-67 with clinicopathologic features and outcome in 103 patients, studied by means of TMA, showed that PR, Ki-67, tumor stage and nodal status were prognostic factors for distant failure in early-stage breast cancer in young patients [18]. Besides these, new interesting associations also resulted from the statistical analysis of our data.

The immunohistochemical evaluation of Akt-1 expression showed a positive association with estrogen receptor expression and an inverse association with tumor grade and nodal metastases, which is in favors of an interpretation of Akt-1 function in estrogen-stimulated growth and cellular differentiation more than in tumor progression. Although these data could appear in contrast with the already reported coexpression of Akt- 1 and HER2 in some cell lines, they are in keeping with the results of Stål et al. [9] reporting a significant frequency of Akt-1 expression in estrogen receptor-positive tumors [19] and with the moderate to high expression of phosphorylated Akt reported in $>70 \%$ of invasive breast carcinoma in a series studied with the microarray technique [20]. The activation of the Akt pathway has been found early in breast cancer, even in the in situ stage [21]. More recently, aberrant Akt-1 signaling has already been related to the blockage of antiestrogen activity implying that steroid-receptor-positive cancers with high Akt-1 signaling might be candidates for antiestrogens in combination with Akt-1 inhibitors [22, 23].

In our study the immunohistochemical expression of Notch-2 was significantly stronger in HER2-positive tu- mors $(++,+++)$ although no significant associations were found with tumor grade or hormone receptor status.

Previous studies have already reported the expression of Notch family molecules in human breast cancers [24, 25] observing higher levels of expression in tumors than in normal breast tissue. The evaluation of Notch-1 and Notch-2 mRNA quantification showed that increased levels of Notch-1 receptor were associated with poorly differentiated tumors while Notch-2 was not significantly related to tumor grade [24]. In the family of Notch receptors, all of which are involved in different stages of tumor progression, Notch-2 acts as a transcriptional and functional regulator of Notch-1 and Notch-3 [26]. Along with other genes, Notch-2 has recently been identified in different tamoxifen-resistant clones [20]. Given the involvement of Notch-2 in cell cycle arrest and cell growth and the possible role of its disrupted form in rendering tumor cells tamoxifen resistant it follows that its immunophenotypic expression can provide useful information about the possible involvement of Notch proteins in breast cancer dynamics.

Although the lack of information regarding both a true quantitative analysis of Notch-2 and Akt-1 expression in our samples and the functional status of these two proteins does not permit us to speculate on the possible mechanisms underlining these relationships, we would like to stress the importance of reporting the association of such molecules with that of histological and phenotypic markers of tumor progression and prognosis. We noted the existence of a conspicuous number of estrogen receptor-positive tumors in which Akt-1 is coexpressed and that show a better degree of differentiation with no lymph node involvement along with the presence of a population of HER2-positive tumors with strong Notch2 expression. In these cases, properly designed studies evaluating the response to tamoxifen- and trastuzumabbased therapies and focusing on the influence of Akt-1 and Notch-2 are definitely needed.

In conclusion, our results further support the role of molecules like Notch and Akt that, although not yet included in treatment strategies for breast cancers, may represent new appealing therapeutic targets for conventional therapy-resistant tumors.

\section{Acknowledgements}

The authors are grateful to Prof. Stefano Pileri for support in TMA construction. This paper was partially supported by the Ricerca Finalizzata Regione Autonoma Sardegna, 2003. 


\section{References}

1 Ries LAG, Harkins D, Krapcho M, Mariotto A, Miller BA, Feuer EJ, et al: SEER Cancer Statistics Review, 1975-2003. Bethesda, National Cancer Institute, 2006.

-2 Goldhirsch A, Glick JH, Gelber RD, Coates AS, Thurlimann B, Senn HJ; Panel members: Meeting highlights: international expert consensus on the primary therapy of early breast cancer 2005. Ann Oncol 2005;16 1569-1583.

3 Nahta R, Yu D, Hung MC, Hortobagyi GN, Esteva FJ: Mechanisms of disease: understanding resistance to HER2-targeted therapy in human breast cancer. Nat Clin Pract Oncol 2006;3:269-280.

-4 Nam Y, Aster JC, Blacklow SC: Notch signaling as a therapeutic target. Curr Opin Chem Biol 2002;6:501-509.

$\checkmark 5$ Kononen J, Bubendorf L, Kallioniemi A, Barlund M, Schraml P, Leighton S, Torhorst J, Mihatsch MJ, Sauter G, Kallioniemi OP: Tissue microarrays for high-throughput molecular profiling of tumor specimens. Nat Med 1998;4:844-847.

$\checkmark 6$ Camp RL, Charette LA, Rimm DL: Validation of tissue microarray technology in breast carcinoma. Lab Invest 2000;80:19431949.

7 Elston CW, Ellis IO: Pathological prognostic factors in breast cancer. I. The value of histological grade in breast cancer: experience from a large study with long-term follow-up. Histopathology 1991;19:403-410.

8 Altman D: Practical Statistics for Medical Research. Boca Raton, Chapman \& Hall/ CRC, 1999.

$>9$ Stål O, Perez-Tenorio G, Akerberg L, Olsson B, Nordenskjold B, Skoog L, Rutqvist LE: Akt kinases in breast cancer and the results of adjuvant therapy. Breast Cancer Res 2003;5: R37-R44.
$>10$ Brennan K, Brown AM: Is there a role for Notch signalling in human breast cancer? Breast Cancer Res 2003;5:69-75.

$>11$ Katoh M, Katoh M: Notch signaling in gastrointestinal tract. Int J Oncol 2007;30:247251.

12 Wang LH, Chan JL, Li W: Rapamycin together with herceptin significantly increased anti-tumor efficacy compared to either alone in ErbB2 overexpressing breast cancer cells. Int J Cancer 2007;121:157-164.

13 Kobayashi S, Kishimoto T, Kamata S, Otsuka M, Miyazaki M, Ishikura H: Rapamycin, a specific inhibitor of the mammalian target of rapamycin, suppresses lymphangiogenesis and lymphatic metastasis. Cancer Sci 2007;98:726-733.

14 Dragowska WH, Verreault M, Yapp DT, Warburton C, Edwards L, Ramsay EC, Huxham LA, Minchinton AI, Gelmon K, Bally MB: Decreased levels of hypoxic cells in gefitinib treated ER(+) HER-2 overexpressing MCF-7 breast cancer tumors are associated with hyperactivation of the mTOR pathway: therapeutic implications for combination therapy with rapamycin. Cancer Res Treat 2007;106:319-331.

15 Chang SB, Miron P, Miron A, Iglehart JD: Rapamycin inhibits proliferation of estrogen-receptor positive breast cancer cells. J Surg Res 2007;138:37-44.

16 Rimm DL, Camp RL, Charette LA, Costa J, Olsen DA, Reiss M: Tissue microarray: a new technology for amplification of tissue resources. Cancer J 2001;7:24-31.

17 Kallioniemi OP, Wagner U, Kononen J, Sauter G: Tissue microarray technology for high-throughput molecular profiling of cancer. Hum Mol Genet 2001;10:657-662.

18 Choi DH, Kim S, Rimm DL, Carter D, Haffty BG: Immunohistochemical biomarkers in patients with early-onset breast carcinoma by tissue microarray. Cancer J 2005;11:404411.
19 Ahmad F, Gao G, Wang LM, Landstrom TR, Degerman E, Pierce JH, Manganiello VC: IL3 and IL-4 activate cyclic nucleotide phosphodiesterases 3 (PDE3) and 4 (PDE4) by different mechanisms in FDCP2 myeloid cells. J Immunol 1999;162:4864-4875.

20 Lin HJ, Hsieh FC, Song H, Lin J: Elevated phosphorylation and activation of PDK-1/ AKT pathway in human breast cancer. $\mathrm{Br}$ J Cancer 2005;93:1372-1381

-21 Bose S, Chandran S, Mirocha JM, Bose N: The Akt pathway in human breast cancer: a tissue-array-based analysis. Mod Pathol 2006;19:238-245.

22 Kurokawa H, Arteaga CL: ErbB (HER) receptors can abrogate antiestrogen action in human breast cancer by multiple signaling mechanisms. Clin Cancer Res 2003;9(1 Pt 2):511S-515S.

23 Perez-Tenorio G, Berglund F, Esguerra Merca A, Nordenskjold B, Rutqvist LE, Skoog L, Stal O: Cytoplasmic p21WAF1/CIP1 correlates with Akt activation and poor response to tamoxifen in breast cancer. Int J Oncol 2006;28:1031-1042.

24 Parr C, Watkins G, Jiang WG: The possible correlation of Notch-1 and Notch-2 with clinical outcome and tumour clinicopathological parameters in human breast cancer. Int J Mol Med 2004;14:779-786.

25 Zarubin T, Jing Q, New L, Han J: Identification of eight genes that are potentially involved in tamoxifen sensitivity in breast cancer cells. Cell Res 2005;15:439-446.

26 Shimizu K, Chiba S, Saito T, Kumano K, Hamada Y, Hirai H: Functional diversity among Notch1, Notch2, and Notch3 receptors. Biochem Biophys Res Commun 2002; 291:775-779. 